\begin{tabular}{lll}
\hline VOL. XXXVIII & 1977 & FASC. 1
\end{tabular}

\title{
ARTINIAN MODULES ARE COUNTABLY GENERATED
}

BY

\author{
D. D. ANDE R ON (BLACKSBURG, VIRGINIA)
}

Let $R$ be a commutative ring with identity. Bass [1] showed that any chain of submodules in a Noetherian $R$-module is countable. The purpose of this note is to prove the dual statement; namely, that any chain of submodules in an Artinian $R$-module is countable. Our general reference will be Lambek [5].

TheOREM. Let $R$ be a commutative ring with identity. Then any Artinian $R$-module $A$ is countably generated.

Proof. Let $S_{n}$ be the $R$-submodule of $A$ generated by all cyclic submodules of length less than or equal to $n$. Then

$$
0=S_{0} \subseteq S_{1} \subseteq S_{2} \subseteq \ldots \text { and } \bigcup_{n=1}^{\infty} S_{n}=A
$$

We show that $\bigcup_{n=1}^{\infty} S_{n}=A$. Let $x \in A$. Then $R x$ being a submodule of $A$ is again Artinian. Now $R x$ and $R / \operatorname{ann}(x)$ are isomorphic as $R$-modules. Since $R$ is commutative, ann $(x)$ is an ideal of $R$, and hence $R / \operatorname{ann}(x)$ is an Artinian ring. By Hopkins' Theorem [3], $R / \operatorname{ann}(x)$ is also Noetherian and hence of finite length. Thus $R x$ has finite length as an $R$-module. Thus $x \in R x \subseteq S_{n}$ for some $n$. To complete the proof it suffices to show that each $S_{n}$ is finitely generated. Now $S_{1}$ is just the socle of $A$, that is, $S_{1}$ is the sum of all simple submodules of $A$. Thus $S_{1}$ is isomorphic to a direct sum of simple modules. Since $S_{1}$ is Artinian (it is a submodule of $A$ ), $\mathbb{S}_{1}$ is a finite direct sum of simple modules, and hence is finitely generated. Assume by induction that $\mathbb{S}_{n}$ is finitely generated. Then $\mathbb{S}_{n+1} / \mathbb{S}_{n}$ is contained in the socle of the Artinian module $A / \mathbb{S}_{n}$. It follows that $\mathbb{S}_{n+1} / \mathbb{S}_{n}$ is finitely generated. Since $S_{n}$ and $S_{n+1} / S_{n}$ are finitely generated, $\mathbb{S}_{n+1}$ is also finitely generated.

The following result is now immediate:

CoRoLlary. Let $R$ be a commutative ring with identity and let $A$ be an Artinian $R$-module. Then any chain of submodules of $A$ is countable. 
Both our result and the result of Bass fail if $R$ is allowed to be noncommutative. Fuchs [2], Example 3, constructs Artinian modules over a polynomial ring in non-commuting indeterminates which have any desired length. In this example there exist cyclic submodules not of finite length.

If $R$ is a left Noetherian ring with identity, then $R$ may have uncountable descending chains of two-sided ideals. For each ordinal $\alpha$, Jategaonkar [4] constructs a local principal left-ideal domain $R$ with $J^{a} \neq 0$, where $J$ is the Jacobson radical of $R$.

\section{REFERENCES}

[1] H. Bass, Descending chains and the Krull ordinal of commutative Noetherian rings, Journal of Pure and Applied Algebra (1971), p. 347-360.

[2] L. Fuchs, Torsion preradicals and ascending Loevoy series of modules, Journal für die reine, und angewandte Mathematik 239-240 (1969), p. 169-179.

[3] C. Hopkins, Rings with minimal condition for left ideals, Annals of Mathematics 40 (1939), p. 712-730.

[4] A. Jategaonkar, A counter-example in ring theory and homological algebra, Journal of Algebra 12 (1969), p. 418-440.

[5] J. Lambek, Lectures on rings and modules, Waltham, Mass., 1966.

Reģu par la Rédaction le 30. 10. 1975;

en version modifiée le 14. 5. 1976 